\title{
On Disjoint Stationary Sets in Topological Spaces
}

\author{
Pralahad Mahagaonkar* \\ Department of Mathematics, Ballari Institute of Technology and Management, Ballari
}

*Corresponding Author: Pralahad Mahagaonkar, Department of Mathematics, Ballari Institute of Technology and Management, Ballari

\begin{abstract}
In this paper we study some new techniques from set theory to general topology and its applications, here we study some the partition relations, cardinal functions and its principals and some more theorems. Further we develop the combinatory of stationary sets we have aimed at person with some knowledge of topology and a little knowledge of set theory.
\end{abstract}

Keywords: Stationary sets, homeomorphic, topologicalspaces, Bairespaces.

\section{INTRODUCTION}

Here we will discuss some interesting subsets of $\omega_{1}-$ sets, Borel subsets, stationary and bi-stationary subsets of $\omega_{1}$ - and some theorems. In addition, we will mention some topological applications of stationary sets . Let us know that $\omega_{1}$ is an uncountable well-ordered set with the special property that if $\alpha<\omega_{1}$, then the initial segment $[0, \alpha)$ of $\omega_{1}$ is countable, and that the countable union of countable sets is countable. Like any linearly ordered set, $\omega_{1}$ has an open interval topology. Le us consider that $\alpha$ is a limit point of the space $\omega_{1}$.

\section{MAIN RESUlTS}

Theorem 1.1 : A Borel subset of $\mathrm{B}(\mathrm{k})$ which is not in $\sigma-L W(<k)$ is a homeomrphic to $\mathrm{B}(\mathrm{k})$.

Definition : $\mathrm{B}(\mathrm{k})$ is the Baire zero dimensional space of weight $\mathrm{k}$, is a function from $\chi \rightarrow k$ with the given $d(f, g)=2^{-n}$, where $\mathrm{n}$ is the least of $f / n \neq g / n$.

Definition: A space ' $\mathrm{X}$ ' is a Baire space iff intersection of countable many dense open subsets of $\mathrm{X}$ is a dense in $X$.

Definition: Any set that intersects every club-set in $\omega_{1}$ is called a stationary subset of $\omega_{1}$.

Definition: Any set $S \subseteq \omega_{1}$ with the property that both $S$ and $\omega_{1}-\mathrm{S}$ intersect every club-set is called a bi-stationary set.

Definition: A topological space $\mathrm{X}$ is said to be metrizable if there exists a metric $\mathrm{d}$ such that $\{\operatorname{Br}(\mathrm{x})$ : $\mathrm{x} \in \mathrm{X}$ and $\mathrm{r}>0$ \} forms a basis generating the topology on $\mathrm{X}$.

Proposition: Any subspace of a metrizable space is metrizable.

Lemma : Let $\mathrm{X}$ is a metrizable space with weight $\mathrm{k}$, where $\mathrm{k}$ is an uncountable regular cardinal, let $\left(d_{\alpha}: \alpha<k\right)$ be a dense subset of X. For $\delta<k$.

Theorem 1.2: There is a subset "s" of $B(w)$ such that both $s$ and $B(k)-S$ meet every subset of $B(w)$ is homeomorphic.

Proof : Let us consider the note that there are $2^{w}$ of $G_{\delta}$ subsets of $\mathrm{B}(\mathrm{w})$ and that each uncountable $G_{\delta}$ has $2^{\omega}$ points. Here we consider the well ordered uncountable $G_{\delta}$ subsets and inductively choose any two points from each one be in $\mathrm{S}$, the other to be in $\mathrm{B}(\mathrm{w})-\mathrm{S}$. The induction continuous because at each step there are less than $2^{\omega}$ points which are chosen so that there are plenty of possible new points.

We would like to construction with $\mathrm{B}(\mathrm{k})$ in the place of $\mathrm{B}(\mathrm{w})$, However, when $k=2^{\omega} \quad$ Then there are $2^{k}$ open sets, but only $\mathrm{k}$ points in the space. 
Theorem 1.3: Let $\mathrm{k}$ be an uncountable regular cardinal, then their $\mathrm{S}$ of $\mathrm{B}(\mathrm{k})$ such that both $\mathrm{S}$ and $\mathrm{B}(\mathrm{k})-\mathrm{S}$ meet every subset of $\mathrm{B}(\mathrm{k})$ is homeomorphic to $\mathrm{B}(\mathrm{k})$.

Proof : Let introduce the new function with $\mathrm{B}(\mathrm{k})$, for $\alpha<k$, Let us consider $\sum_{\alpha} \alpha=\{\alpha: \alpha$ is a function, dom $\alpha \in \omega$, ran $\sigma \subset \alpha\}$ For $\sigma \in \sum_{k} \alpha$ define for $\alpha=\{f \in B(k): \sigma \subset f\}$. Such that for each $[\alpha]$ is closed and open subset of $\mathrm{B}(\mathrm{k})$, and $\left\{[\sigma]: \sigma \in \sum_{i}\right\}$ is a base for $\mathrm{B}(\mathrm{k})$,for $\mathrm{B}(\mathrm{k})$ define $f^{*}=$ Sup ran $f$ for $\sigma \in \sum_{k}$ define $\sigma^{*}=$ Sup $\operatorname{ran} \sigma$. Note that for each $\alpha<k,\left\{\sigma: \sigma^{*}<\alpha\right\}<k$. By using the above Lemma it is clear that $A \subset C F \omega \cap k$ such that both A and CF of $C F \omega \bigcap k$ are stationary sets in k, Thus z satisfies the conclusion of Theorem 1.3 .

Theorem 1.4: There are two Baire spaces whose product is not a Baire.

Proof : Let $\mathrm{A}$ and $\mathrm{B}$ are any disjoint stationary sub sets of $\omega_{1}$. Further consider $X=\left\{f \in B\left(\omega_{1}\right): f^{*} \in A\right\}: Y=\left\{g \in B\left(\omega_{1}\right): g^{*} \in B\right\}$, for every dense of $G_{\delta}$ of $\mathrm{B}(\mathrm{k})$ is homeomorphic to $\mathrm{B}(\mathrm{k})$, so by Definition, $\mathrm{X}$ any $\mathrm{Y}$ are Baire spaces.

For $n \in \omega$, let $U_{n}=\left\{(f, g) \in X \times Y: f(n)<g^{*}\right.$ and $\left.g(n)<f^{*}\right\}$.It is to verify that $\mathrm{U}_{\mathrm{n}}$ is dense and open subset of XxY. Thus the Contradiction.

Theorem 1.5: Suppose there is a continuous injective mapping $\mathrm{h}: \mathrm{S} \rightarrow \mathrm{T}$ where $\mathrm{S}$ and $\mathrm{T}$ are stationary sets. Then $\mathrm{S} \cap \mathrm{T}$ is also stationary.

Proof: Because there is a continuous injective mapping from $S$ to $T$, then $S-T$ cannot be stationary. But $\mathrm{S}=(\mathrm{S} \cap \mathrm{T}) \cup(\mathrm{S}-\mathrm{T})$ is stationary so that the set $\mathrm{S} \cap \mathrm{T}$ must be stationary.

\section{REFERENCES}

[1] Balough, Z. and Rudin, M.E., Monotonic normality, Topology and its Applications 47(1992), 115-127.

[2] Buzyakova, R., Injections into function spaces over ordinals, Topology and its Applications 157(2010), 2844-2849.

[3] Buzyakova, R., and Vural, C., Stationary sets in topological and paratopological groups, Houston Journal of Mathematics 40(2014), 267-273.

[4] Devlin, K., The Joy of Sets, Springer-Verlag, New York, 1993.

[5] Van Douwen, E., and Lutzer, D. On the classification of stationary sets, Michigan Mathematics Journal 26(1979), 47-64.

[6] Engelking, R., General Topology, Heldermann Verlag, Berlin, 1989.

[7] Halmos, P., Measure Theory, van Nostrand, New York, 1950.

[8] Heath, R., Lutzer, D., and Zenor, P., Monotonically normal spaces, Transactions of the American Mathematical Society, 178 (1973), 481-493.

[9] Kunen, K., Set Theory: An Introduction to Independence Proofs, North Holland, Amsterdam, 1980.

[10] Oxtoby, J., Measure and Category, Springer-Verlag, New York, 1971.

[11] Royden, H., Real Analysis, Macmillan, New York,1963. [15] Rudin, M., A subset of the countable ordinals, American Mathematical Monthly 64(1957), 351-2.

[12] Munkres, James R., “Topology: A First Course”, Prentice-Hall, Inc., Englewood Cliffs, N.J., 1975.

[13] Kunen, Kenneth, "Set Theory. An Introduction to Independence Proofs", Studies in Logic and the Foundations of Mathematics, 102, North-Holland Publishing Co., Amsterdam-New York, 1980.

[14] Engelking, R.; Lutzer, D. Paracompactness in ordered spaces. Fund. Math. 94 (1977), no. 1, 49-58.

[15] Fleissner, W. G.; Kunen, K. Barely Baire spaces. Fund. Math. 101 (1978), no. 3, 229- 240

Citation: Pralahad Mahagaonka, " On Disjoint Stationary Sets in Topological Spaces ", International Journal of Scientific and Innovative Mathematical Research (IJSIMR), vol. 7, no. 9, pp. 1-2, 2019. Available: DOI: http://dx.doi.org/10.20431/2347-3142.0709001

Copyright: (C) 2019 Authors. This is an open-access article distributed under the terms of the Creative Commons Attribution License, which permits unrestricted use, distribution, and reproduction in any medium, provided the original author and source are credited. 